\title{
Two-loop Resummation in Fractional Analytic Perturbation Theory
}

\author{
Alexander P. Bakulev ${ }^{a *}$ \\ a Bogoliubov Laboratory of Theoretical Physics, JINR \\ Jolio-Curie 6, Dubna, 141980 Russia
}

November 22, 2018

\begin{abstract}
This talk describes the resummation approach in (Fractional) Analytic Perturbation Theory (FAPT) in QCD. First, we make a short historical review of the (F)APT approach and then shortly describe the global scheme of FAPT which allows one to take into account heavy-quark thresholds. After that we show how it is possible to resum a non-power series in (F)APT both in the one- and two-loop approximations. As an application we suggest our analysis of the Higgs boson decay $H^{0} \rightarrow b \bar{b}$, important for the LHC program, and of the vector-current Adler function.
\end{abstract}

\section{Analytic Perturbation Theory in QCD}

First, I'd like to remind the history of Analytic Perturbation Theory (APT). Strictly speaking, APT was initiated by the paper of N. N. Bogolyuov et al. in 1959 [1], where ghost-free effective coupling for QED was constructed using the dispersion-relations method. Then, in 1982, Radyushkin [2], and Krasnikov and Pivovarov [3], using the same dispersion-relations technique, suggested regular (for $s \geq \Lambda^{2}$ ) QCD running coupling in the Minkowski region, $\alpha_{s}(s) \equiv a_{s}(s) / \beta_{f}$, namely, $a_{s}[L]=\pi^{-1} \arctan (\pi / L)$ with $L=\ln \left(s / \Lambda^{2}\right)$ and $\beta_{f}=b_{0}\left(N_{f}\right) /(4 \pi)=$ $\left(11-2 N_{f} / 3\right) /(4 \pi)\left[1\right.$ (This normalized coupling $a_{s}[L]$ in standard QCD PT is just $1 / L$ in the one-loop approximation.)

Thirteen years later, Dokshitzer et al. constructed an IR finite $\alpha_{s}^{\mathbf{e f f}}\left(Q^{2}\right)$ in the Euclidean domain using a renormalon-based approach with dispersion relations [4. After that, Jones and Solovtsov [5, 6] discovered the coupling which appears to be finite for all $s$ and coincides with the Radyushkin's one for $s \geq \Lambda^{2}$, namely

$$
\mathfrak{A}_{1}[L]=\int_{s}^{\infty} \frac{\rho_{1}(\sigma)}{\sigma} d \sigma=\frac{1}{\pi} \arccos \frac{L}{\sqrt{\pi^{2}+L^{2}}} .
$$

Just at the same time, Ball, Beneke and Braun reproduced in the pQCD-based renormalon approach $a_{s}[L]=\pi^{-1} \arctan (\pi / L)$ in the low-energy Minkowski region [7, 8], and Shirkov and Solovtsov [9], using the same dispersion-relations approach of [1], discovered the ghost-free coupling,

$$
\mathcal{A}_{1}[L]=\int_{0}^{\infty} \frac{\rho_{1}(\sigma)}{\sigma+Q^{2}} d \sigma=\frac{1}{L}-\frac{1}{e^{L}-1}
$$

\footnotetext{
${ }^{*}$ E-mail: bakulev@theor.jinr.ru

${ }^{1}$ We use the notations $f\left(Q^{2}\right)$ and $f[L]$ in order to specify the arguments we mean - squared momentum $Q^{2}$ or its logarithm $L=\ln \left(s / \Lambda^{2}\right)$ or $\ln \left(Q^{2} / \Lambda^{2}\right)$, that is $f[L]=f\left(\Lambda^{2} \cdot e^{L}\right)$ and $\Lambda^{2}$ is usually referred to the $N_{f}=3$ region.
} 
in the Euclidean region with $L=\ln \left(Q^{2} / \Lambda^{2}\right)$. But Shirkov-Solovtsov approach, named APT, appears to be more powerful: in the Euclidean domain, $-q^{2}=Q^{2}, L=\ln Q^{2} / \Lambda^{2}$, it generates the set of images for the effective coupling and its $n$-th powers, $\left\{\mathcal{A}_{n}[L]\right\}_{n \in \mathbb{N}}$, whereas in the Minkowski domain, $q^{2}=s, L=\ln s / \Lambda^{2}$, it generates another set, $\left\{\mathfrak{A}_{n}[L]\right\}_{n \in \mathbb{N}}$ (see also in [10]). APT is based on the Renormalization Group (RG) and causality that guarantees standard perturbative UV asymptotics and spectral properties. The power series $\sum_{m} d_{m} a_{s}^{m}[L]$ transforms into a non-power series $\sum_{m} d_{m} \mathfrak{A}_{m}[L]$ or $\sum_{m} d_{m} \mathcal{A}_{m}[L]$ in APT. The global version of APT, which takes into account the heavy-quark thresholds, has been elaborated in [11.

By the analytization in APT for an observable $f\left(Q^{2}\right)$ we mean the "Källen-Lehmann" representation

$$
\left[f\left(Q^{2}\right)\right]_{\mathrm{an}}=\int_{0}^{\infty} \frac{\rho_{f}(\sigma)}{\sigma+Q^{2}-i \epsilon} d \sigma
$$

with $\rho_{f}(\sigma)=\frac{1}{\pi} \mathbf{I m}[f(-\sigma)]$, which transforms to different prescriptions for couplings in the Euclidean and Minkowski domains

$$
\begin{aligned}
& \mathcal{A}_{n}[L]=\mathbf{A}_{\mathbf{E}}\left[\alpha_{s}^{n}\right]=\int_{0}^{\infty} \frac{\rho_{n}(\sigma)}{\sigma+Q^{2}} d \sigma ; \\
& \mathfrak{A}_{n}[L]=\mathbf{A}_{\mathbf{M}}\left[\alpha_{s}^{n}\right]=\int_{s}^{\infty} \frac{\rho_{n}(\sigma)}{\sigma} d \sigma .
\end{aligned}
$$

In the one-loop approximation, we have then $\rho_{1}(\sigma)=1 / \sqrt{L_{\sigma}^{2}+\pi^{2}}$ and Eqs. (11), whereas analytic images of the higher powers $(n \geq 2, n \in \mathbb{N})$ are [2, ?]

$$
\left(\begin{array}{l}
\mathcal{A}_{n}[L] \\
\mathfrak{A}_{n}[L]
\end{array}\right)=\frac{1}{(n-1) !}\left(-\frac{d}{d L}\right)^{n-1}\left(\begin{array}{l}
\mathcal{A}_{1}[L] \\
\mathfrak{A}_{1}[L]
\end{array}\right) .
$$

At first glance, the APT is a complete theory supplying tools to produce an analytic answer for any perturbative series in QCD. But in 2001 Karanikas and Stefanis reminded the need for more tools to produce analytic expressions for hadronic observables, calculated perturbatively [12. Indeed, in the standard QCD PT one has also:

(i) the factorization procedure in QCD that gives rise to the appearance of logarithmic factors of the type: $a_{s}^{\nu}[L] L$;

(ii) the RG evolution that generates evolution factors of the type $B\left(Q^{2}\right)=\left[Z\left(Q^{2}\right) / Z\left(\mu^{2}\right)\right]$ $B\left(\mu^{2}\right)$, which reduce in the one-loop approximation to $Z\left(Q^{2}\right) \sim a_{s}^{\nu}[L]$ with $\nu=\gamma_{0} /\left(2 b_{0}\right)$ being a fractional number (here the subscript 0 means that the corresponding quantity is calculated in the one-loop approximation).

They suggested the principle of analytization "as a whole" in the $Q^{2}$ plane for hadronic amplitudes, obtained in QCD PT, thus generalizing the analytic approach suggested in [13.2 A review of both approaches can be found in [14, whereas an extensive discussion of the APT application to the pion form factor analysis is contained in [15].

All that means that in order to generalize APT in the "analytization as a whole" direction one needs to construct analytic images of new functions: $a_{s}^{\nu}, a_{s}^{\nu} L^{m}, \ldots$ This task has been performed in the framework of the so-called FAPT, suggested in [16, 17]. Now we briefly

\footnotetext{
${ }^{2}$ More precisely, they proposed the analytization recipe for terms like $\int_{0}^{1} d x \int_{0}^{1} d y \alpha_{\mathrm{s}}\left(Q^{2} x y\right) f(x) f(y)$, which can be treated as an effective account for the logarithmic terms in the next-to-leading-order approximation of perturbative QCD.
} 
describe this approach. In the one-loop approximation, using the recursive relation (3), we can obtain explicit expressions for $\mathcal{A}_{\nu}[L]$ and $\mathfrak{A}_{\nu}[L]$ :

$$
\begin{aligned}
\mathcal{A}_{\nu}[L] & =\frac{1}{L^{\nu}}-\frac{F\left(e^{-L}, 1-\nu\right)}{\Gamma(\nu)} ; \\
\mathfrak{A}_{\nu}[L] & =\frac{\sin \left[(\nu-1) \arccos \left(\frac{L}{\sqrt{\pi^{2}+L^{2}}}\right)\right]}{\pi(\nu-1)\left(\pi^{2}+L^{2}\right)^{(\nu-1) / 2}} .
\end{aligned}
$$

Here $F(z, \nu)$ is the reduced Lerch transcendental function, which is an analytic function in $\nu$. This function has very interesting properties, which were discussed extensively in the previous papers [16, 17, 18, 19].

The construction of FAPT with a fixed number of quark flavors, $N_{f}$, is a two-step procedure: we start with the perturbative result $\left[a_{s}\left(Q^{2}\right)\right]^{\nu}$, generate the spectral density using $\rho_{\nu}(\sigma)=$ $\frac{1}{\pi} \mathbf{I m}\left[a_{s}(-\sigma)\right]^{\nu}$, and then obtain analytic couplings $\mathcal{A}_{\nu}[L]$ and $\mathfrak{A}_{\nu}[L]$ via the relations like Eqs. (2). Here $N_{f}$ is fixed and factorized out. We can proceed in the same manner with $N_{f}$-dependent quantities: $\left[\alpha_{s}\left(Q^{2} ; N_{f}\right)\right]^{\nu} \Rightarrow \bar{\rho}_{\nu}\left(\sigma ; N_{f}\right)=\bar{\rho}_{\nu}\left[L_{\sigma} ; N_{f}\right] \equiv \rho_{\nu}(\sigma) / \beta_{f}^{\nu} \Rightarrow \overline{\mathcal{A}}_{\nu}\left[L ; N_{f}\right]$ and $\overline{\mathfrak{A}}_{\nu}\left[L ; N_{f}\right]$ - here $N_{f}$ is fixed, but not factorized out. The global version of FAPT [18, which takes into account heavy-quark thresholds, is constructed along the same lines but starting from the global perturbative coupling $\left[\alpha_{s}^{\text {glob }}\left(Q^{2}\right)\right]^{\nu}$, being a continuous function of $Q^{2}$ due to adopting different values of QCD scales $\Lambda_{f}$, which correspond to different values of $N_{f}$. We illustrate here the case of only one heavy-quark threshold at $s=m_{4}^{2}$, corresponding to the transition $N_{f}=3 \rightarrow N_{f}=4$. Then we obtain the discontinuous spectral density

$$
\rho_{n}^{\text {glob }}(\sigma)=\theta\left(L_{\sigma}<L_{4}\right) \bar{\rho}_{n}\left[L_{\sigma} ; 3\right]+\theta\left(L_{4} \leq L_{\sigma}\right) \bar{\rho}_{n}\left[L_{\sigma}+\lambda_{4} ; 4\right]
$$

with $L_{\sigma} \equiv \ln \left(\sigma / \Lambda_{3}^{2}\right), L_{f} \equiv \ln \left(m_{f}^{2} / \Lambda_{3}^{2}\right)$ and $\lambda_{f} \equiv \ln \left(\Lambda_{3}^{2} / \Lambda_{f}^{2}\right)$ for $f=4$, which is expressed in terms of fixed-flavor spectral densities with 3 and 4 flavors, $\bar{\rho}_{n}[L ; 3]$ and $\bar{\rho}_{n}\left[L+\lambda_{4} ; 4\right]$. However, it generates the continuous Minkowski coupling

$$
\mathfrak{A}_{\nu}^{\text {glob }}[L]=\theta\left(L<L_{4}\right)\left(\overline{\mathfrak{A}}_{\nu}[L ; 3]+\Delta_{43} \overline{\mathfrak{A}}_{\nu}\right)+\theta\left(L_{4} \leq L\right) \overline{\mathfrak{A}}_{\nu}\left[L+\lambda_{4} ; 4\right]
$$

with $\Delta_{43} \overline{\mathfrak{A}}_{\nu}=\overline{\mathfrak{A}}_{\nu}\left[L_{4}+\lambda_{4} ; 4\right]-\overline{\mathfrak{A}}_{\nu}\left[L_{4} ; 3\right]$ and the analytic Euclidean coupling $\mathcal{A}_{\nu}^{\text {glob }}[L]$

$$
\mathcal{A}_{\nu}^{\text {glob }}[L]=\overline{\mathcal{A}}_{\nu}\left[L+\lambda_{4} ; 4\right]+\int_{-\infty}^{L_{4}} \frac{\bar{\rho}_{\nu}\left[L_{\sigma} ; 3\right]-\bar{\rho}_{\nu}\left[L_{\sigma}+\lambda_{4} ; 4\right]}{1+e^{L-L_{\sigma}}} d L_{\sigma} .
$$

The same strategy one needs to use when working in a higher-loop approximation - then, analytic formulas even for the fixed- $N_{f}$ case are unavailable (the spectral density is expressed in terms of the imaginary part of the Lambert function) and one is forced to use integral representations of the type (2) to calculate analytic couplings and other quantities of interest. One more complication here is the more complex evolution law: for example, in the two-loop approximation the evolution factor $B\left(Q^{2}\right)=\left[Z\left(Q^{2}\right) / Z\left(\mu^{2}\right)\right]$ has $Z\left(Q^{2}\right) \sim a_{s}^{\nu_{0}}[L]\left(1+c_{1} a_{s}[L]\right)^{\nu_{1}}$, with exponents $\nu_{0}$ and $\nu_{1}$ being known numerical coefficients — for more details see in [18].

\section{Resummation in (F)APT}

We consider now the perturbative expansion of a typical physical quantity, like the Adler function and the ratio $R$, in the one-loop APT. Due to the limited space of our presentation, we provide all formulas only for quantities in the Minkowski region:

$$
\mathcal{R}[L]=\sum_{n=1}^{\infty} d_{n} \mathfrak{A}_{n}[L] .
$$


We suggest that there exist a generating function $P(t)$ for the coefficients $\tilde{d}_{n}=d_{n} / d_{1}$ :

$$
\tilde{d}_{n}=\int_{0}^{\infty} P(t) t^{n-1} d t \quad \text { with } \quad \int_{0}^{\infty} P(t) d t=1 .
$$

To shorten our formulae, we use for the integral $\int_{0}^{\infty} f(t) P(t) d t$ the following notation: $\langle\langle f(t)\rangle\rangle_{P(t)}$. Then, the coefficients $d_{n}=d_{1}\left\langle\left\langle t^{n-1}\right\rangle\right\rangle_{P(t)}$ and the series (7a) can be represented as follows (we put for shortness $d_{1}=1$ ):

$$
\mathcal{R}[L]=\sum_{n=1}^{\infty}\left\langle\left\langle t^{n-1}\right\rangle\right\rangle_{P(t)} \mathfrak{A}_{n}[L]
$$

\section{$2.1 \quad$ One-loop case}

Due to the recurrence relations (44), as has been shown in [20], we have the exact result for the sum in (17c), viz.,

$$
\mathcal{R}[L]=\left\langle\left\langle\mathfrak{A}_{1}[L-t]\right\rangle\right\rangle_{P(t)} .
$$

The integral over the variable $t$ has a rigorous meaning, ensured by the finiteness of the coupling $\mathfrak{A}_{1}[t] \leq 1$ and the fast fall-off of the generating function $P(t)$. This result resembles the result of resummation of the large $\left(\beta_{0}^{n} \alpha_{s}\right)^{n-1}$ terms due to Neubert [21], but here we have no problem with renormalon poles, i. e., in our case the integral $\left\langle\left\langle\mathfrak{A}_{1}[L-t]\right\rangle\right\rangle_{P(t)}$ is nicely convergent. Note that the same type of approach in the one-loop APT has been independently invented two years later 3 by Cvetic and Valenzuela 22 within the framework of the so-called "skeleton expansion".

We have first accomplished the generalization of result (8) to the case of global APT [11, taking into account heavy-quark thresholds [18, 23]. Then, one starts with the series of type $(7 \mathrm{c})$, where $\mathfrak{A}_{n}[L]$ are substituted by their global analogs $\mathfrak{A}_{n}^{\text {glob }}[L]$ (note that due to the different normalizations of the global couplings, $\mathfrak{A}_{n}^{\text {glob }}[L] \simeq \mathfrak{A}_{n}[L] / \beta_{f}$, the coefficients $d_{n}$ should also be changed). Then, we have

$$
\mathcal{R}^{\text {glob }}[L]=\theta\left(L<L_{4}\right)\left\langle\left\langle\Delta_{4} \overline{\mathfrak{A}}_{1}[t]+\overline{\mathfrak{A}}_{1}\left[L-\frac{t}{\beta_{3}} ; 3\right]\right\rangle\right\rangle_{P(t)}+\theta\left(L \geq L_{4}\right)\left\langle\left\langle\overline{\mathfrak{A}}_{1}\left[L+\lambda_{4}-\frac{t}{\beta_{4}} ; 4\right]\right\rangle\right\rangle_{P(t)},
$$

where $\Delta_{4} \overline{\mathfrak{A}}_{\nu}[t] \equiv \overline{\mathfrak{A}}_{\nu}\left[L_{4}+\lambda_{4}-t / \beta_{4} ; 4\right]-\overline{\mathfrak{A}}_{\nu}\left[L_{3}-t / \beta_{3} ; 3\right]$.

The second generalization has been obtained for the case of global FAPT. The starting point in this case is the generalized recurrence relation

$$
-\frac{1}{n+\nu} \frac{d}{d L} \mathcal{F}_{n+\nu}[L]=\mathcal{F}_{n+1+\nu}[L]
$$

where $\mathcal{F}[L]$ denotes one of the analytic quantities $\mathcal{A}[L]$ or $\mathfrak{A}[L]$. The result of summation of the series $\sum_{n=0}^{\infty}\left\langle\left\langle t^{n-1}\right\rangle\right\rangle_{P(t)} \mathfrak{A}_{n+\nu}^{\text {glob }}[L]$ is the complete analog of Eq. (9) with the substitutions

$$
P(t) \Rightarrow P_{\nu}(t)=\int_{0}^{1} P\left(\frac{t}{1-x}\right) \frac{\nu x^{\nu-1} d x}{1-x}
$$

where $d_{0} \Rightarrow d_{0} \overline{\mathfrak{A}}_{\nu}[L], \overline{\mathfrak{A}}_{1}[L-t] \Rightarrow \overline{\mathfrak{A}}_{1+\nu}[L-t]$, and $\Delta_{4} \overline{\mathfrak{A}}_{1}[t] \Rightarrow \Delta_{4} \overline{\mathfrak{A}}_{1+\nu}[t]$. All needed formulas have also been obtained in parallel for the Euclidean case, see in [18].

\footnotetext{
${ }^{3}$ Note that the paper of Mikhailov [20] was put into arXiv in November, 2004, and after a long discussions with referees published only in 2007, whereas the paper of Cvetic and Valenzuela 22] was put in arXiv in August, 2006, and published the same year. It is interesting to remind here the title of Mikhailov's paper: "Generalization of BLM procedure and its scales in any order of $p Q C D:$ A practical approach" — we see that it was devoted to completely different subject and Eq. (8), numerated in this paper as Eq. (2.7), was obtained in passing.
} 


\subsection{Two-loop case}

In the case of the two-loop running of $\alpha_{s}^{(2)}\left(Q^{2}\right)=4 \pi a_{(2)}\left(Q^{2}\right) / b_{0}$, i. e., when the $\beta$-function has the following form (with $c_{1} \equiv b_{1} / b_{0}^{2}$ )

$$
\beta\left(a_{(2)}\right)=-a_{(2)}^{2}\left[1+c_{1} a_{(2)}\right],
$$

we have two types of complication. First, the recurrence relations (3) and (10) transform into

$$
-\frac{1}{n+\nu} \frac{d}{d L} \mathcal{F}_{n+\nu}[L]=\mathcal{F}_{n+1+\nu}[L]+c_{1} \mathcal{F}_{n+2+\nu}[L],
$$

where $\mathcal{F}[L]$ denotes now one of the analytic quantities $\mathcal{A}^{(2)}[L], \mathfrak{A}^{(2)}[L]$, or $\rho^{(2)}[L]$. Second, the evolution factors appear to be more complicated as well, namely,

$$
\mathcal{Z}_{\nu_{0} ; \nu_{1}}[L]=a_{(2)}^{\nu_{0}}[L]\left(1+c_{1} a_{(2)}\right)^{\nu_{1}}[L]
$$

with exponents $\nu_{0}$ and $\nu_{1}$ being numerical combinations of anomalous-dimension coefficients $\gamma_{0}$, $\gamma_{1}$, and $\beta$-function coefficients $\beta_{0}, \beta_{1}$.

For these reasons, we need to consider the resummation of the following series:

$$
\begin{aligned}
\mathcal{S}_{\nu}[L] & =\sum_{n=1}^{\infty}\left\langle\left\langle t^{n-1}\right\rangle\right\rangle_{P(t)} \mathcal{F}_{n+\nu}[L] ; \\
\mathcal{S}_{\nu_{0}, \nu_{1}}[L] & =\sum_{n=1}^{\infty}\left\langle\left\langle t^{n-1}\right\rangle\right\rangle_{P(t)} \mathcal{F}_{n+\nu_{0}, \nu_{1}}[L] .
\end{aligned}
$$

In the last line, one has $\mathcal{F}_{n+\nu_{0}, \nu_{1}}[L]=\mathcal{B}_{n+\nu_{0}, \nu_{1}}^{(2)}[L]$ or $\mathfrak{B}_{n+\nu_{0}, \nu_{1}}^{(2)}[L]$ (or -in the case of global FAPT - the spectral density $\rho_{n+\nu_{0}, \nu_{1}}^{(2)}[L]$ for these functions) with

$$
\left(\begin{array}{c}
\mathcal{B}_{\nu_{0} ; \nu_{1}}[L] \\
\mathfrak{B}_{\nu_{0} ; \nu_{1}}[L]
\end{array}\right)=\left(\begin{array}{c}
\mathbf{A}_{\mathbf{E}} \\
\mathbf{A}_{\mathbf{M}}
\end{array}\right)\left[a_{(2)}^{\nu_{0}}\left(1+c_{1} a_{(2)}\right)^{\nu_{1}}\right][L]
$$

being the analytic images of the two-loop evolution factors in the Euclidean and Minkowski regions, correspondingly. These problems have been successfully resolved in our recent paper [24] and here we show only the results.

First, we need to define the two-loop evolution "logarithmic time" $\tau(t)$ :

$$
\tau(t) \equiv t-c_{1} \ln \left[1+\frac{t}{c_{1}}\right] .
$$

Then the two-loop FAPT resummation procedure produces the following answers:

For the series (15a) the resummed expression is

$$
\begin{aligned}
\mathcal{S}_{\nu}[L] & =\left\langle\left\langle\mathcal{F}_{1+\nu}[L]-\frac{t^{2}}{c_{1}+t} \int_{0}^{1} z^{\nu} d z \dot{\mathcal{F}}_{1+\nu}[L+\tau(t z)-\tau(t)]\right\rangle_{P(t)}\right. \\
& +\left\langle\left\langle\frac{c_{1} t}{c_{1}+t}\left\{\mathcal{F}_{2+\nu}[L]-\int_{0}^{1} d z \frac{t^{2} z^{\nu+1}}{c_{1}+t z} \dot{\mathcal{F}}_{2+\nu}[L+\tau(t z)-\tau(t)]\right\}\right\rangle\right\rangle_{P(t)},
\end{aligned}
$$

whereas for the series (15b) -

$$
\begin{aligned}
\mathcal{S}_{\nu_{0}, \nu_{1}}[L] & =\left\langle\left\langle\mathcal{B}_{1+\nu_{0} ; \nu_{1}}[L]+\delta_{0, \nu_{0}+\nu_{1}} t\left[\frac{c_{1}}{c_{1}+t}\right]^{1-\nu_{1}} \mathcal{B}_{2+\nu_{0} ; \nu_{1}}[L-\tau(t)]\right\rangle\right\rangle_{P(t)} \\
& -\left\langle\left\langle\frac{t^{2}}{\left(c_{1}+t\right)^{1-\nu_{1}}} \int_{0}^{1} d z \frac{z^{\nu_{0}+\nu_{1}}}{\left(c_{1}+t z\right)^{\nu_{1}}} \frac{d \mathcal{B}_{1+\nu_{0} ; \nu_{1}}[L+\tau(t z)-\tau(t)]}{d L}\right\rangle\right\rangle_{P(t)} \\
& +\left\langle\left\langle\frac{c_{1} t}{\left(c_{1}+t\right)^{1-\nu_{1}}} \int_{0}^{1} d z \frac{\left(\nu_{0}+\nu_{1}\right) z^{\nu_{0}+\nu_{1}-1}}{\left(c_{1}+t z\right)^{\nu_{1}}} \mathcal{B}_{2+\nu_{0} ; \nu_{1}}[L+\tau(t z)-\tau(t)]\right\rangle\right\rangle_{P(t)},
\end{aligned}
$$


with $\delta_{0, \nu_{0}+\nu_{1}}$ being a Kronecker delta symbol.

\section{Applications to Higgs boson decay}

Here we analyze the Higgs boson decay to a $\bar{b} b$ pair. For its width we have

$$
\Gamma(\mathrm{H} \rightarrow b \bar{b})=\Gamma_{0}^{b}\left(m_{b}^{2}\right) \frac{\widetilde{R}_{\mathrm{S}}\left(M_{H}^{2}\right)}{3 m_{b}^{2}},
$$

with $\Gamma_{0}^{b}\left(m_{b}^{2}\right)=3 G_{F} M_{H} m_{b}^{2} / 4 \sqrt{2} \pi, m_{b}$ and $M_{H}$ are the pole mass of the $b$-quark and the mass of the Higgs boson, respectively, $\widetilde{R}_{\mathrm{S}}\left(M_{H}^{2}\right)=\bar{m}_{b}^{2}\left(M_{H}^{2}\right) R_{\mathrm{S}}\left(M_{H}^{2}\right)$ and $R_{\mathrm{S}}(s)$ is the $R$-ratio for the scalar correlator, see for details in [16, 25].

In the one-loop FAPT this generates the following non-power series expansion 4

$$
\widetilde{\mathcal{R}}_{\mathrm{S}}^{(1)}[L]=3 \hat{m}_{(1)}^{2}\left\{\mathfrak{A}_{\nu_{0}}^{\text {glob }}[L]+d_{1}^{\mathrm{S}} \sum_{n \geq 1} \frac{\tilde{d}_{n}^{\mathrm{S}}}{\pi^{n}} \mathfrak{A}_{n+\nu_{0}}^{\text {glob }}[L]\right\},
$$

where $\hat{m}_{(1)}^{2}=8.53 \pm 0.09 \mathrm{GeV}^{2}$ is the RG-invariant of the one-loop $\bar{m}_{b}^{2}\left(\mu^{2}\right)$ evolution, written in the following way: $\bar{m}_{b}^{2}\left(\mu^{2}\right)=\hat{m}_{(1)}^{2} \alpha_{s}^{\nu_{0}}\left(\mu^{2}\right)$ with $\nu_{0}=2\left[\gamma_{0} / b_{0}\right]_{N_{f}=5}=1.04$, and $\gamma_{0}$ is the leading-order quark-mass anomalous dimension. This value $\hat{m}_{(1)}^{2}$ has been obtained using the one-loop relation [26, 27] between the pole mass of the $b$ quark, $m_{b}$, and the value of the running mass at the scale $\mu_{*}=\bar{m}_{b}\left(\mu_{*}^{2}\right)$, which we call $\bar{m}_{b}\left(\bar{m}_{b}^{2}\right)$. Here we also extract the value of $d_{1}^{\mathrm{S}}=17 C_{\mathrm{F}} / 4=17 / 3$ out of higher perturbative coefficients, so that $\tilde{d}_{n}^{\mathrm{S}}=3 d_{n}^{\mathrm{S}} / 17$ and $\tilde{d}_{1}^{\mathrm{S}}=1$.

In the two-loop case we obtain

$$
\begin{aligned}
& \bar{m}_{b}^{2}\left(\mu^{2}\right)=\hat{m}_{(2)}^{2} \alpha_{s}^{\nu_{0}}\left(\mu^{2}\right)\left[1+\frac{c_{1} b_{0} \alpha_{s}\left(\mu^{2}\right)}{4 \pi^{2}}\right]^{\nu_{1}} ; \\
& \widetilde{\mathcal{R}}_{\mathrm{S}}^{(2)}[L]=3 \hat{m}_{(2)}^{2}\left\{\mathfrak{B}_{\nu_{0}, \nu_{1}}^{(2) \text {;lob }}[L]+d_{1}^{\mathrm{S}} \sum_{n \geq 1} \frac{\tilde{d}_{n}^{\mathrm{S}}}{\pi^{n}} \mathfrak{B}_{n+\nu_{0}, \nu_{1}}^{(2) ; \text {;lob }}[L]\right\},
\end{aligned}
$$

where the RG-invariant mass $\hat{m}_{(2)}^{2}=8.22 \pm 0.09 \mathrm{GeV}^{2}$, while the value of $\nu_{0}$ is the same as in the one-loop case, and $\nu_{1}=2\left[\left(\gamma_{1} b_{0}-\gamma_{0} b_{1}\right) /\left(b_{0} b_{1}\right)\right]_{N_{f}=5}=1.86\left(\gamma_{1}\right.$ is the next-to-leading-order quark-mass anomalous dimension). Note that we determined both values, $\hat{m}_{(1)}^{2}$ and $\hat{m}_{(2)}^{2}$, using the estimates for $\bar{m}_{b}\left(\bar{m}_{b}^{2}\right)$ derived by Penin and Steinhauser [28].

In order to estimate now the importance of higher-order corrections, we need to construct some model for the generating function $P(t)$. We use a Lipatov-like model [23] with $c=2.4$, $\beta=-0.52$, which generates factorially growing coefficients $\tilde{d}_{n}^{\mathrm{s}}$ :

$$
\begin{aligned}
\tilde{d}_{n}^{\mathrm{S}} & =c^{n-1} \frac{\Gamma(n+1)+\beta \Gamma(n)}{1+\beta}, \\
P_{\mathrm{S}}(t) & =\frac{(t / c)+\beta}{c(1+\beta)} e^{-t / c} .
\end{aligned}
$$

this model gives a very good prediction for $\tilde{d}_{n}^{\mathrm{S}}$ with $n=2,3,4$, calculated in QCD PT [25]: 7.50, 61.1 , and 625 in comparison with 7.42,62.3, and 620. Moreover, it predicts the value $\tilde{d}_{5}^{\mathrm{S}}=7826$ which is in a good agreement with the PMS [29, 30] prediction $\tilde{d}_{5}^{\text {PMS }}=7782$, obtained in [24].

Then, we apply the FAPT resummation technique to estimate how good is the $N$ th order truncation

$$
\Gamma_{H \rightarrow b \bar{b}}^{(1)}[L ; N]=\Gamma_{0}^{b}\left(\hat{m}_{(1)}^{2}\right)\left[\mathfrak{A}_{\nu_{0}}^{\text {glob }}[L]+d_{1}^{\mathrm{S}} \sum_{n=1}^{N} \frac{\tilde{d}_{n}^{\mathrm{S}}}{\pi^{n}} \mathfrak{A}_{n+\nu_{0}}^{\text {glob }}[L]\right]
$$

\footnotetext{
${ }^{4}$ Appearance of denominators $\pi^{n}$ in association with the coefficients $\tilde{d}_{n}$ is due to $d_{n}$ normalization.
} 
in approximating the whole one-loop width $\Gamma_{H \rightarrow b \bar{b}}^{(1)}[L]$ in the range $L \in[12.4,13.5]$ which corresponds to the range $M_{H} \in[100,172] \mathrm{GeV}$ with $\Lambda_{\mathrm{QCD}}^{N_{f}=3}=201 \mathrm{MeV}$ and $\mathfrak{A}_{1}^{\text {glob }}\left(m_{Z}^{2}\right)=0.1226$. In this range, we have $L_{5}<L<L_{6}=\ln \left(m_{t}^{2} / \Lambda_{3}^{2}\right)$ and hence

$$
\Gamma_{H \rightarrow b \bar{b}}^{(1) ; \infty}[L]=\Gamma_{0}^{b}\left(\hat{m}_{(1)}^{2}\right)\left\{\mathfrak{A}_{\nu_{0}}^{\text {glob }}[L]+\frac{d_{1}^{S}}{\pi}\left\langle\left\langle\overline{\mathfrak{A}}_{1+\nu_{0}}\left[L+\lambda_{5}-\frac{t}{\pi \beta_{5}} ; 5\right]+\Delta_{6} \overline{\mathfrak{A}}_{1+\nu_{0}}\left[\frac{t}{\pi}\right]\right\rangle_{P_{\nu_{0}}^{\mathrm{S}}}\right\}\right.
$$

with $P_{\nu_{0}}^{\mathrm{S}}(t)$ defined via Eqs. (11) and (21). We analyze the accuracy of the truncated FAPT expressions (22a ) and compare them with the total sum (22b) using relative errors $\Delta_{N}[L]=$ $1-\Gamma_{H \rightarrow b \bar{b}}^{(1)}[L ; N] / \Gamma_{H \rightarrow b \bar{b}}^{(1) ; \infty}[L]$. In Fig. 1 we show these errors for $N=2, N=3$, and $N=4$ in the analyzed range of $L \in[11,13.7]$. We see that already $\Gamma_{H \rightarrow b \bar{b}}^{(1)}[L ; 2]$ gives an accuracy of the order of $2.5 \%$, whereas $\Gamma_{H \rightarrow b \bar{b}}^{(1)}[L ; 3]$ is of the order of $1 \%$. Looking in the left panel of Fig. 1, we
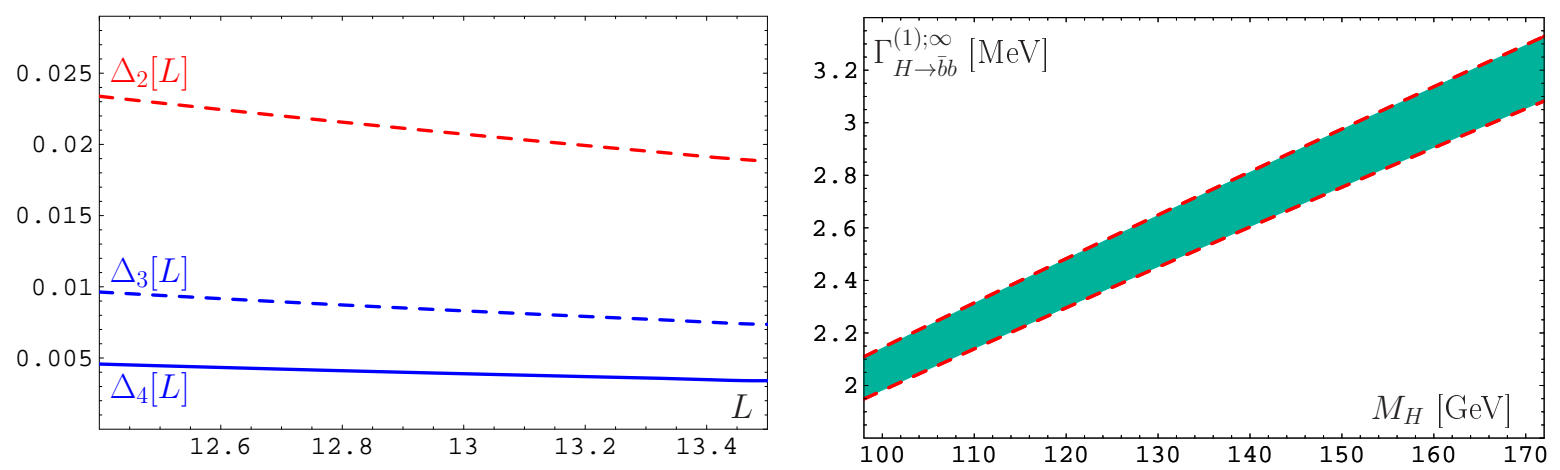

Figure 1: Left panel: The relative errors $\Delta_{N}[L], N=2,3$ and 4, of the truncated FAPT in comparison with the exact summation result, Eq. (22b). Right panel: The width $\Gamma_{H \rightarrow b \bar{b}}$ as a function of the Higgs boson mass $M_{H}$ in the resummed FAPT. The width of the shaded strip is due to the overall uncertainties, induced by the uncertainties of the resummation procedure and the pole mass error-bars. Both panels show the results obtained in the one-loop FAPT [24].

understand that in order to have an accuracy better than $0.5 \%$, we need to take into account the 4 -th correction. We also verified that the uncertainty due to $P(t)$-modelling is small, $\lesssim 0.6 \%$, while the $\bar{m}_{b}\left(\bar{m}_{b}^{2}\right)$-induced uncertainty is of the order of $2 \%$. The overall uncertainty then is of the order of $3 \%$, see in the right panel of Fig. 1.

Qualitatively, the same picture is reproduced at the two-loop order [24]. In the left panel of Fig. 2, we discuss the convergence properties of the decay widths, truncated at the order $N$, relative to the resummed two-loop result $\Gamma_{H \rightarrow b \bar{b}}^{(2) ; \infty}\left(M_{H}\right)$. We see that our conclusions drawn from the one-loop analysis remain valid. Indeed, $\Gamma_{H \rightarrow b \bar{b}}^{(2)}\left(M_{H} ; 2\right)$ deviates from the resummed quantity by not more than $2 \%$, whereas $\Gamma_{H \rightarrow b \bar{b}}^{(2)}\left(M_{H} ; 3\right)$ reaches an even higher precision level of the order of $0.7 \%$.

In the right panel of Fig. 2, we show the results for the decay width $\Gamma_{H \rightarrow b \bar{b}}^{\infty}\left(M_{H}\right)$ in the resummed two-loop FAPT, varying the mass in the interval $\hat{m}_{(2)}=8.22 \pm 0.13 \mathrm{GeV}$ according to the Penin-Steinhauser estimate $\bar{m}_{b}\left(\bar{m}_{b}^{2}\right)=4.35 \pm 0.07 \mathrm{GeV}$ [28]. Comparing this outcome with the one-loop result - upper strip in the same panel of this figure - reveals a $5 \%$ reduction of the two-loop estimate. This reduction consists of two parts: one part $(\approx+7 \%)$ is due to the difference $\hat{m}_{(1)}^{2}-\hat{m}_{(2)}^{2}$ in both approximations, while the other $(\approx-2 \%)$ comes from the difference in the values of $R_{S}\left(M_{H}\right)$ in the one- and the two-loop approximations. 

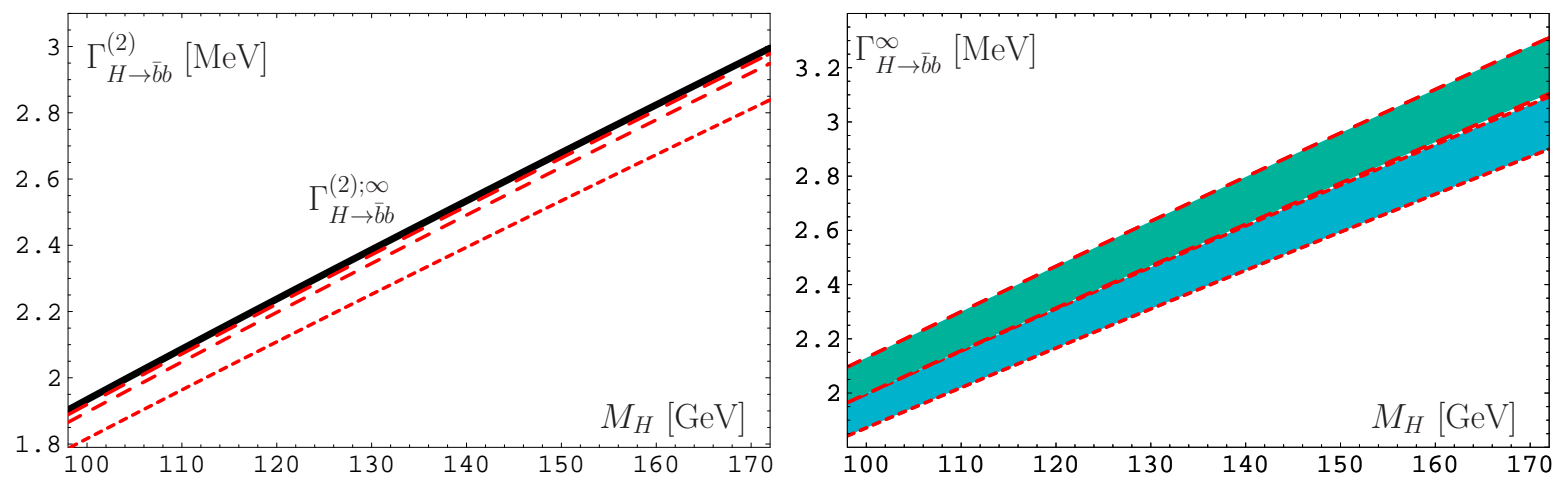

Figure 2: Left panel: The two-loop width $\Gamma_{H \rightarrow b \bar{b}}$ as a function of the Higgs-boson mass $M_{H}$ in the resummed (black solid line) and the truncated (at the order $N$ ) FAPT [24]. Here, the short-dashed line corresponds to $N=1$, the dashed one to $N=2$, and the long-dashed line to $N=3$. Right panel: The two-loop width $\Gamma_{H \rightarrow b \bar{b}}^{\infty}$ as a function of the Higgs-boson mass $M_{H}$ in the resummed FAPT is shown (lower strip). The upper strip shows the corresponding one-loop result.

\section{Application to the vector correlator Adler function}

Now we analyze the Adler function of the vector correlator. We model the generating function of the perturbative coefficients $d_{n}$ [31] (note that here $d_{1}=1$, so that $\tilde{d}_{n}=d_{n}$ )

$$
D_{\mathrm{V}}[L]=d_{0}+\sum_{n=1}^{\infty} d_{n}\left(\frac{\alpha_{s}[L]}{\pi}\right)^{n}
$$

using a generalized Lipatov-like model:

$$
\begin{aligned}
d_{n}^{\mathrm{V}} & =c^{n-1} \frac{\delta^{n+1}-n}{\delta^{2}-1} \Gamma(n), \\
P_{\mathrm{V}}(t) & =\frac{\delta e^{-t / c \delta}-(t / c) e^{-t / c}}{c\left(\delta^{2}-1\right)} .
\end{aligned}
$$

Our predictions, obtained with this generating function by fitting the two known coefficients $\tilde{d}_{2}$ and $\tilde{d}_{3}$, have been included in Table 1 (see 24]). We observe a good agreement between our estimate $d_{4}^{\mathrm{V}}=27.1$ and the value 27.4 , calculated recently by Chetyrkin et al. in Ref. [31. Would we use instead a fitting procedure, which would take into account the fourth-order coefficient $d_{4}$ in order to predict $d_{5}$, we should readjust the model parameters in (24) to the new values $\{c=3.5526, \delta=1.32453\}$. In order to explore to what extent the exact knowledge of the higher-order coefficients $d_{n}$ is important, we employed our model (24) in [24] with different values of the parameters: $c=3.63$ and $\delta=1.3231$. These values were selected in order to reproduce the exact value of the coefficient $d_{4}=27.4$ by the value, close to the Naive NonAbelization (NNA) prediction [32]. The difference between the resummed results of the two models in the region of $N_{f}=4$ appears to be very small — of the order of $0.2 \%$. We also show in Table 1 the $d_{n}$ estimates obtained by using the Improved NNA (INNA) approximation, developed in our last paper [24] by taking into account not only the $b_{0}^{n} \alpha_{s}^{n}$ terms, but also the $b_{1}^{k} b_{0}^{n-2 k} \alpha_{s}^{n}$ ones. This technique produces predictions for higher-order coefficients in terms of intervals for the $d_{n}$ values. We see that our model predictions for the $d_{5}$ coefficient are all inside the INNA interval. 
Table 1: Coefficients $d_{n}$ for the Adler-function series with $N_{f}=4$. The numbers in the square brackets denote the lower and the upper limits of the Improved NNA (INNA) estimates.

\begin{tabular}{|c|ccccc|}
\hline \hline PT coefficients & $d_{1}$ & $d_{2}$ & $d_{3}$ & $d_{4}$ & $d_{5}$ \\
\hline \hline pQCD results with $N_{f}=4[31]$ & 1 & 1.52 & 2.59 & 27.4 & - \\
\hline \hline Model (24) with $c=3.555, \delta=1.3245$ & 1 & 1.52 & 2.59 & 27.1 & 2024 \\
\hline Model (24) with $c=3.553, \delta=1.3245$ & 1 & 1.52 & 2.60 & 27.3 & 2025 \\
\hline Model (24) with $c=3.630, \delta=1.3231$ & 1 & 1.53 & 2.26 & 20.7 & 2020 \\
\hline "INNA" prediction of [24] & 1 & 1.44 & {$[3.5,9.6]$} & {$[20.4,48.1]$} & {$[674,2786]$} \\
\hline \hline
\end{tabular}

Then, we have

$$
\begin{aligned}
D_{\mathrm{V}}^{\mathrm{APT}}\left(Q^{2}\right) & =1+\sum_{n \geq 1} \frac{d_{n}^{\mathrm{V}}}{\pi^{n}} \mathcal{A}_{n}^{\text {glob }}\left(Q^{2}\right), \\
D_{\mathrm{V}}^{\mathrm{APT}}\left(Q^{2} ; N\right) & =1+\sum_{n=1}^{N} \frac{d_{n}^{\mathrm{V}}}{\pi^{n}} \mathcal{A}_{n}^{\text {glob }}\left(Q^{2}\right) .
\end{aligned}
$$

The global-APT resummation result for $D_{\mathrm{V}}^{\mathrm{APT}}\left(Q^{2}\right)$ can be estimated using our resummation formulas from [18, 23, 24], which we shortly explained in Sect. 2, We show in Fig. 3 the relative errors $\Delta_{N}^{\mathrm{V}}\left(Q^{2}\right)=1-D_{\mathrm{V}}^{\mathrm{APT}}\left(Q^{2} ; N\right) / D_{\mathrm{V}}^{\mathrm{APT}}\left(Q^{2}\right)$ evaluated in the range $Q^{2} \in[2,20] \mathrm{GeV}^{2}$ for four values $N=1,2,3,4$. We observe from this figure that already $D_{\mathrm{V}}^{\mathrm{APT}}\left(Q^{2} ; 1\right)$ provides an accuracy in the vicinity of $1 \%$, whereas $D_{\mathrm{V}}^{\mathrm{APT}}\left(Q^{2} ; 2\right)$ is smaller then $0.1 \%$ in the interval $Q^{2}=1-20 \mathrm{GeV}^{2}$. This means that there is no real need to calculate further corrections: at the level of an accuracy of the order of $1 \%$ it is quite enough to take into account only the terms up to $d_{2}$. Again, we verified that this conclusion is stable with respect to the variation of the model $P_{\mathrm{V}}(t)$ parameters. This conclusion is in some sense surprising: The best order of truncation of the FAPT series in the region $Q^{2}=2-20 \mathrm{GeV}^{2}$ is reached by the $\mathrm{N}^{2} \mathrm{LO}$ approximation, i.e., by keeping just the $d_{2}$-term.

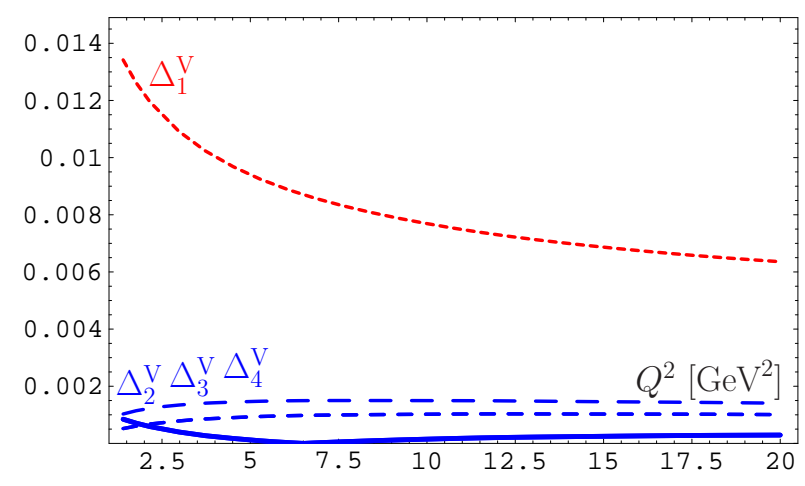

Figure 3: The relative errors $\Delta_{N}^{\mathrm{V}}\left(Q^{2}\right)$ evaluated for different values of $N: N=1$ (short-dashed red line), $N=2$ (solid blue line), $N=3$ (dashed blue line), and $N=4$ (long-dashed blue line) of the truncated APT given by Eq. (25b), in comparison with the exact result of the APT resummation procedure. 


\section{Conclusions}

We described here the resummation approach in the global versions of both APT and FAPT for the one- and two-loop running of the effective QCD coupling. We argued that this approach produces finite answers, provided the generating function $P(t)$ of the perturbative coefficients $d_{n}$ is known. We considered two applications of our approach to the physical problems of current interest.

In the case of the Higgs boson decay, $H \rightarrow \bar{b} b$, the main conclusion is that to achieve a truncation accuracy of the order of $1 \%$ for the width $\Gamma_{H \rightarrow b \bar{b}}\left(M_{H}\right)$ it is enough to take into account up to the third correction - in complete agreement with Kataev\&Kim [33]. In the case of the vector-current Adler function, $\mathcal{D}_{\mathrm{V}}\left(Q^{2}\right)$, the truncation accuracy is of the order $0.1 \%$, already reached at $\mathrm{N}^{2} \mathrm{LO}$, i. e., by taking into account up to the second-order correction.

In our approach the knowledge of higher-order coefficients, $d_{4}$, is needed in order to construct a reliable model for the generating functions $P(t)$. Meanwhile, we showed that the exact values of still higher coefficients, $d_{5}$, are not so important.

\section{Acknowledgements}

I wish to thank Sergey Mikhailov and Nico Stefanis for a fruitful collaboration, and Konstantin Chetyrkin, Andrey Grozin, Andrey Kataev and Alexey Pivovarov for useful remarks and discussions. This work was supported in part by the Russian Foundation for Fundamental Research, grant No. 08-01-00686, the BRFBR-JINR Cooperation Programme, contract No. F10D-001, and the Heisenberg-Landau Programme under Grant 2010.

\section{References}

[1] N. N. Bogolyubov, A. A. Logunov, and D. V. Shirkov, Soviet Physics JETP 10 (1960) 574.

[2] A. V. Radyushkin, JINR Rapid Commun. 78 (1996) 96; [JINR Preprint, E2-82-159, 26 Febr. 1982; arXiv: hep-ph/9907228].

[3] N. V. Krasnikov and A. A. Pivovarov, Phys. Lett. B116 (1982) 168.

[4] Y. L. Dokshitzer, G. Marchesini, and B. R. Webber, Nucl. Phys. B469, 93 (1996).

[5] H. F. Jones and I. L. Solovtsov, Phys. Lett. B349, 519 (1995).

[6] H. F. Jones, I. L. Solovtsov, and O. P. Solovtsova, Phys. Lett. B357, 441 (1995).

[7] M. Beneke and V. M. Braun, Phys. Lett. B348 (1995) 513.

[8] P. Ball, M. Beneke, and V. M. Braun, Nucl. Phys. B452 (1995) 563.

[9] D. V. Shirkov and I. L. Solovtsov, JINR Rapid Commun. 2 [76] (1996) 5; Phys. Rev. Lett. 79 (1997) 1209; Theor. Math. Phys. 150 (2007) 132.

[10] Y. A. Simonov, Phys. Atom. Nucl. 65 (2002) 135.

[11] D. V. Shirkov, Theor. Math. Phys. 127, 409 (2001); Eur. Phys. J. C22, 331 (2001).

[12] A. I. Karanikas and N. G. Stefanis, Phys. Lett. B504 (2001) 225; B636, 330 (2006).

[13] N. G. Stefanis, W. Schroers, and H.-C. Kim, Phys. Lett. B449 (1999) 299; Eur. Phys. J. C18 (2000) 137.

[14] N. G. Stefanis, Lect. Notes Phys. 616 (2003) 153. 
[15] A. P. Bakulev, K. Passek-Kumerički, W. Schroers, and N. G. Stefanis, Phys. Rev. D70 (2004) 033014.

[16] A. P. Bakulev, S. V. Mikhailov, and N. G. Stefanis, Phys. Rev. D72 (2005) 074014, 119908(E); ibid. 75 (2007) 056005; 77 (2008) 079901(E).

[17] A. P. Bakulev, A. I. Karanikas, and N. G. Stefanis, Phys. Rev. D72 (2005) 074015.

[18] A. P. Bakulev Phys. Elem. Part. Nucl. 40 (2009) 715.

[19] N. G. Stefanis, arXiv:0902.4805 [hep-ph].

[20] S. V. Mikhailov, JHEP 06 (2007) 009 hep-ph/0411397.

[21] M. Neubert, Phys. Rev. D51 (1995) 5924.

[22] G. Cvetic and C. Valenzuela, Phys. Rev. D74 (2006) 114030 hep-ph/0608256].

[23] A. P. Bakulev and S. V. Mikhailov, in Proc. Int. Seminar on Contemp. Probl. of Part. Phys., dedicated to the memory of I. L. Solovtsov, Dubna, Jan. 17-18, 2008., Eds. A. P. Bakulev et al. (JINR, Dubna, 2008), pp. 119-133 (arXiv:0803.3013).

[24] A. P. Bakulev, S. V. Mikhailov, and N. G. Stefanis, JHEP 1006 (2010) 085.

[25] P. A. Baikov, K. G. Chetyrkin, and J. H. Kühn, Phys. Rev. Lett. 96 (2006) 012003.

[26] K. G. Chetyrkin and M. Steinhauser, Phys. Rev. Lett. 83, 4001 (1999).

[27] K. G. Chetyrkin and M. Steinhauser, Nucl. Phys. B573 (2000) 617.

[28] A. A. Penin and M. Steinhauser, Phys. Lett. B538 (2002) 335.

[29] A. L. Kataev and V. V. Starshenko, Mod. Phys. Lett. A10 (1995) 235.

[30] K. G. Chetyrkin, B. A. Kniehl, and A. Sirlin, Phys. Lett. B402 (1997) 359.

[31] P. A. Baikov, K. G. Chetyrkin, and J. H. Kühn, Phys. Rev. Lett. 101 (2008) 012002; ibid. 104 (2010) 132004.

[32] D. J. Broadhurst and A. L. Kataev, Phys. Lett. B315 (1993) 179; ibid. B544 (2002) 154.

[33] A. L. Kataev and V. T. Kim, in Proc. Int. Seminar on Contemp. Probl. of Part. Phys., dedicated to the memory of I. L. Solovtsov, Dubna, Jan. 17-18, 2008., Eds. A. P. Bakulev et al. (JINR, Dubna, 2008), pp. 167-182 (arXiv:0804.3992);

PoS ACAT08 (2009) 004. 